\title{
A geometrical point of view on linearized beta-deformations
}

\author{
Andrei Mikhailov ${ }^{\dagger}$ and Segundo P. Milián \\ Instituto de Física Teórica, Universidade Estadual Paulista \\ R. Dr. Bento Teobaldo Ferraz 271, Bloco II - Barra Funda \\ CEP:01140-070 - São Paulo, Brasil
}

\begin{abstract}
It is known that the supermultiplet of beta-deformations of $\mathcal{N}=4$ supersymmetric Yang-Mills theory can be described in terms of the exterior product of two adjoint representations of the superconformal algebra. We present a super-geometrical interpretation of this fact, by evaluating the deforming operator on some special coherent states in the space of supersingletons. We also discuss generalization of this approach to other finite-dimensional deformations of the $\mathcal{N}=4$ supersymmetric Yang-Mills theory.
\end{abstract}

$\dagger$ on leave from Institute for Theoretical and Experimental Physics, ul. Bol. Cheremushkinskaya, 25, Moscow 117259, Russia 


\section{Contents}

1 Introduction 1

2 Harmonic superspace 6

2.1 Twistors and Grassmannian . . . . . . . . . . . . . . 6 6

2.2 Berezinian and some notations . . . . . . . . . . . . 7

2.3 Action of $G L(4 \mid 4, \mathbf{R})$ transformations on twistors . . . . . . . 8

2.4 Solutions of free classical super-Maxwell equations from twistor space ....................... . . 8

3 Coherent wavefunctions in free theory 12

4 Beta deformation of the free Yang-Mills action $\quad 13$ 4.1 Definition of $\delta_{Z_{(1)}, Z_{(2)}}(Z) \ldots \ldots \ldots \ldots$

4.2 Orientation of $L_{\mathrm{rd}}$ and discontinuity of $\delta_{Z_{(1)}, Z_{(2)}}^{\xi} \ldots \ldots \ldots$

4.3 Symmetry of $\delta_{Z_{(1)}, Z_{(2)}}^{\xi}(Z) \ldots \ldots \ldots \ldots$

4.4 The case when $\xi \wedge \xi$ corresponds to zero deformation . . . . . 17

4.5 Deformation of the form $\int \phi \psi \psi \ldots \ldots . . . . . . . .19$

5 Other finite-dimensional representations 21

A Measure defined by an odd linear operator 23

B Some integrals $\quad 24$

\section{Introduction}

AdS/CFT correspondence has been under vigorous study for more than 20 years, bringing important results. Historically the first application was to compare the BPS (or "protected") states of the $N=4$ supersymmetric YangMills theory to the solutions of Type IIB supergravity (SUGRA) linearized near the "background solution" $A d S_{5} \times S^{5}$. Both are representations of Lie super-algebra $\mathbf{p s u}(2,2 \mid 4)$. The fact that they precisely match has been firmly established, see [1] and references therein. However, the structure of these representations has not been sufficiently well studied. We understand well those representations which are unitary. Those are irreducible, generated by highest weight vectors (UIR) [2]. But not all interesting representations are 
irreducible (and even for UIRs, it would be good to have several alternative descriptions). For example, there are finite-dimensional representations and, to the best of our knowledge, it is not clear if they are irreducible or not ${ }^{1}$. Understanding, from various points of view, the structure of the representations appearing in AdS/CFT would be useful. In particular, it is needed for constructing the string worldsheet massless vertex operators, along the lines of $[3,4,5,6]$. (The observation of [3] was that, to construct the vertex, it is enough to know the structure of the representation of $\mathbf{p s u}(2,2 \mid 4)$ in which the corresponding state transforms.) And an efficient construction of massless vertex operators would, in turn, allow the computation of the SUGRA scattering amplitudes on $A d S_{5} \times S^{5}$ in the pure spinor formalism [7].

In this paper we will suggest a point of view on the finite-dimensional represenations. The idea is to probe them by evaluating the corresponding deformations of the free Yang-Mills action on certain solutions of free field equations. The result is some generalized function of the parameters of those free solutions. This generalized function is manifestly supersymmetric and has a nice super-geometrical interpretation. We discuss mostly the case of the so-called beta-deformation [8]. The structure of the corresponding representation is known from the supergravity analysi: ${ }^{2}[9]$. Here we make this structure transparent on the field theory side. Then we apply our method to other finite-dimensional representations. Under the assumption (unproven) that they are irreducible, we identify them with particular Young diagramms.

We will now proceed to describing our results.

Deformations transforming in finite-dimensional representations Consider single trace deformations of $\mathcal{N}=4$ supersymmetric Yang-Mills theory (SYM):

$$
S_{\mathrm{SYM}} \mapsto S_{\mathrm{SYM}}+\varepsilon \int d^{4} x U
$$

where $U$ is a single-trace operator. According to AdS/CFT [1, they correspond to some deformations of the Type IIB superstring theory on $A d S_{5} \times S^{5}$. Let us restrict ourselves to infinitesimal deformations, i.e. compute only to the first order in the deformation parameter $\varepsilon$. Besides $\varepsilon$, there are two other

\footnotetext{
${ }^{1}$ finite dimensional representations of Lie superalgebras are not necessarily semisimple

${ }^{2}$ Beta-deformations were discovered in [8], but their structure as representations of $\operatorname{psu}(2,2 \mid 4)$ was not studied. It was computed using AdS/CFT in [9].
} 
parameters: the Yang-Mills coupling constant $g_{Y M}$ and the number of colors $N$. In this paper, we will consider $N$ being very large. As for the $g_{Y M}$, there are two opposite limits: the limit $g_{Y M}=0$ of free $\mathcal{N}=4$ super Yang-Mills theory (SYM) and the strong coupling limit. In the weak coupling limit we can do perturbative calculations in SYM, and in the strong coupling limit we can use the superstring theory on the classical supergravity (SUGRA) background $A d S_{5} \times S^{5}$.

Both SYM theory and superstring on $A d S_{5} \times S^{5}$ are invariant under the superconformal group $P S U(2,2 \mid 4)$, and it is natural to ask how the deformations of the form (1) transform under this group. In particular, some deformations of the form (1) transform in finite-dimensional representations 4]. As an example of a finite-dimensional representation, consider $U$ of Eq. (1) of the form [4]:

$$
\int d^{4} x \operatorname{tr}\left(\Phi_{1}+i \Phi_{2}\right)^{n+4}+\text { c.c. }
$$

where $\Phi_{1}, \ldots, \Phi_{6}$ are the scalar fields of the $\mathcal{N}=4$-super-Yang-Mills theory. Consider the linear space of all deformations obtained from Eq. (2) by acting with all possible polynomials of generators of $p s u(2,2 \mid 4)$. In the free theory, this gives a finite-dimensional representation. Let us call it $E_{n+4}^{\text {free }}$.

Most of finite-dimensional deformations of the free theory combine into infinite-dimensional representations in the quantum interacting theory. But $E_{n+4}^{\text {free }}$ stays finite-dimensional. We will call it just $E_{n+4}$. This follows from the finiteness of the operator $\operatorname{tr}\left(\Phi_{1}+i \Phi_{2}\right)^{n+4}$ and its descendants. Finiteness implies that the conformal transformations of this operators are same in quantum theory as in classical theory (no countertems $\Rightarrow$ no anomalous dimension). Therefore, the subspace generated by acting on Eq. (2) with the conformal generators is the same in classical and quantum theory. In particular, it is finite-dimensional. To generate the full representation, it remains to act with supersymmetry and superconformal generators. However, those are nilpotent modulo elements of $s u(2,2) \oplus s u(4)$, and therefore cannot change the property of the representation being finite-dimensional.

We will now argue that $E_{n+4}$ is isomorphic to the representation $E_{n+4}^{\text {free }}$ which is generated by acting with $p s u(2,2 \mid 4)$ on Eq. (2) in the free $\mathcal{N}=4$ super-Yang-Mills theory. Indeed, let us consider the chiral primary representation, which is defined as the infinite-dimensional representation generated by the local operator $\operatorname{tr}\left(\Phi_{1}+i \Phi_{2}\right)^{n+4}(0)$ by acting on it with $p s u(2,2 \mid 4)$. This is a unitary representation. Let us call it $F_{n+4}$. Similarly, there is a $F_{n+4}$ 
free version $F_{n+4}^{\text {free }}$ which is defined in the same way as $F_{n+4}$, but in the free theory. We start by pointing out that $F_{n+4}$ is isomorphic to $F_{n+4}^{\text {free }}$, because both are highest weight unitary irreducible representations of $p s u(2,2 \mid 4)$ with the same highest weight $\operatorname{tr}\left(\Phi_{1}+i \Phi_{2}\right)^{n+4}(0)$. On the other hand, our finitedimensional representations $E_{n+4}$ and $E_{n+4}^{\text {free }}$ are fully determined by $F_{n+4}$ and $F_{n+4}^{\text {free }}$, respectively, in the following way. Elements of $E_{n+4}$ are of the form $\int d^{4} x \rho(x) \mathcal{O}(x)$ where $\mathcal{O}$ is an element of $F_{n+4}$ (a local operator), but inserted at the point $x$ instead of 0 . When we act on $\int d^{4} x \rho(x) \mathcal{O}(x)$ with an element of $p s u(2,2 \mid 4)$, the result is determined by how this element acts on $\mathcal{O}(x)$, i.e. by the structure of $F_{n+4}$. The isomorphism $F_{n+4} \stackrel{\simeq}{\longrightarrow} F_{n+4}^{\text {free }}$ implies a map $\mathcal{O} \mapsto \mathcal{O}^{\text {free }}$ commuting with the action of $p s u(2,2 \mid 4)$. This establishes an isomoprhism $E_{n+4} \stackrel{\simeq}{\longrightarrow} E_{n+4}^{\text {free }}$.

Still, it is not clear to us if $E_{n+4}$ is irreducible or not. If it is irreducible, then considerations of [4] and Section 5 of this paper suggest that it should correspond to the supersymmetric Young diagramms (see Section 5 and [4] and references there):
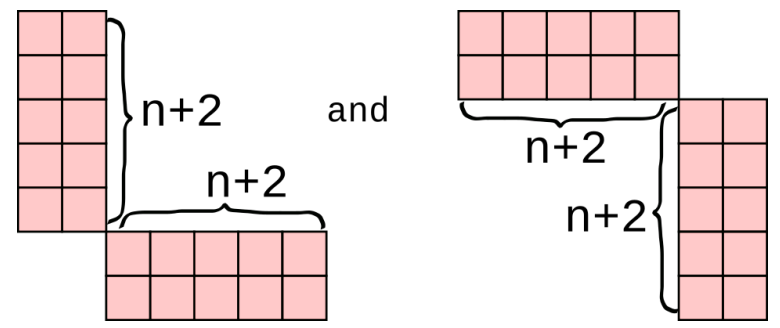

Beta-deformation In this letter we will mostly consider a particular example of a finite-dimensional representation: the linearized $\beta$-deformation [8, 9]. In some sense it corresponds to $n=-1$, but the construction is different. If we literally take $n=-1$ in Eq. (2), and start acting by the generators of $p s u(2,2 \mid 4)$, we obtain an infinite-dimensional representation. However, there is a finite dimensional subrepresentation, which is generated by:

$$
\int d^{4} x \epsilon^{\alpha \beta} Q_{\alpha}^{\left[-\frac{1}{2}, \frac{1}{2}, \frac{1}{2}\right]} Q_{\beta}^{\left[-\frac{1}{2}, \frac{1}{2}, \frac{1}{2}\right]} \operatorname{tr}\left(\Phi_{1}+i \Phi_{2}\right)^{3}+\text { c.c. }
$$

where $Q_{\alpha}^{\left[-\frac{1}{2}, \frac{1}{2}, \frac{1}{2}\right]}$ are supersymmetry generators with weight $\left[-\frac{1}{2}, \frac{1}{2}, \frac{1}{2}\right]$ under the elements $\Phi_{1} \frac{\partial}{\partial \Phi_{2}}-\Phi_{2} \frac{\partial}{\partial \Phi_{1}}, \Phi_{3} \frac{\partial}{\partial \Phi_{4}}-\Phi_{4} \frac{\partial}{\partial \Phi_{3}}$ and $\Phi_{5} \frac{\partial}{\partial \Phi_{6}}-\Phi_{6} \frac{\partial}{\partial \Phi_{5}}$ of the $s u(4)$ Cartan subalgebra. This deformation is preserved by $Q_{\alpha}^{\left[-\frac{1}{2}, \frac{1}{2}, \frac{1}{2}\right]}$ and $Q_{\dot{\alpha}}^{\left[\frac{1}{2},-\frac{1}{2},-\frac{1}{2}\right]}$. It starts with the cubic term, but also has quartic terms. In the language 
of $\mathcal{N}=1$ superspace, the cubic terms come from $\delta W^{\prime \prime} \psi \psi$ and quartic terms come from $\left|(W+\delta W)^{\prime}\right|^{2}$ terms, where $W$ is the superpotential of the $\mathcal{N}=4$ Yang-Mills in the $\mathcal{N}=1$ notations, and $\delta W$ its Leigh-Strassler deformation. Notice that the quartic terms are proportional to $g_{\mathrm{YM}}$. In the free field limit the quartic terms are absent, but the cubic terms survive and define a deformation of the free Yang-Mills theory.

It was found on the AdS side that the supermultiplet of $\beta$-deformations is related to the wedge product of two copies of the adjoint representation of $\mathbf{g}=\operatorname{psu}(2,2 \mid 4)[9]:$

$$
\frac{(\mathrm{g} \wedge \mathrm{g})_{0}}{\mathrm{~g}}
$$

This is an irreducible representation. The subscript 0 imposes the constraint of zero internal commutator; the origin of this constraing on the AdS side was explained in [10, 11, 12]. The geometrical origin of $\mathbf{g} \wedge \mathbf{g}$ is immediately visible on the AdS side; the corresponding vertex operator is essentially the wedge product of two global symmetry currents on the string worldsheet. But it is not immediately obvious why such structure would turn up on the field theory side. In the free field limit the deforming operator $U$ is some quibic expression in elementary fields. It was shown in [13] that these expressions transform in the representation given by Eq. (5). In this letter we will present a geometrical construction making this result more transparent. We will use twistor methods. First in Section 2 we will review the formalism of Harmonic superspace, and how it describes classical solutions of free theory. We then introduce in Section 3 some special coherent states, which are $\delta$-functions with support on $\alpha$-planes. (The ideas of Sections 2 and 3 are well known to experts, but we could not find a reference with explicit formulas suitable for our needs.) In Section 4 we consider the evaluation of the beta-deformation term $\int d^{4} x U$ in Eq. (1) on the formal sum of coherent states, also known as "perturbiner". For our approach it is essential to work in space-time signature $2+2$. In this case the evaluation gives a well-defined generalized function of the parameters of our coherent states. We explicitly describe this generalized function and study its properties. In Section 5 we conjecture a similar description for the deformations of the form Eq. (2) with $n \geq 0$.

It should be possible to drive this results using twistor string theory [14, 15], from a generalization of the results of $]^{3}[16,17,18$. Notice that twistor string

\footnotetext{
${ }^{3}$ We would like to thank the Referee for pointing out to us these references
} 
theory requires complex twistor suprspace $\mathbf{C P}^{3 \mid 4}$, while for our considerations here real twistors $\mathbf{R P}^{3 \mid 4}$ are enough ( $c p$. Section 3.2 of [14]).

Our approach can be interpreted as classifying the possible deformations of the action by looking at their effect on the scattering amplitudes. In fact, Eqs. (53) and (91) can be interpreted as the deformation of the $k$-point scattering amplitude at the minimal value of $k$ for which the deformation is nonzero. Conceptually similar approach was used in [19] for classification of counterterms in supergravity.

\section{Harmonic superspace}

\subsection{Twistors and Grassmannian}

We will work with the space-time signature $2+2$. With this signature the superconformal group is $\operatorname{PSL}(4 \mid 4, \mathbf{R})$. Following [20] we will think about the four-dimensional $\mathcal{N}=4$ superspace as a Grassmannian manifold $M=$ $\operatorname{Gr}\left(2 \mid 2, \mathbf{R}^{4 \mid 4}\right)$, which parametrizes subspaces $\mathbf{R}^{2 \mid 2} \subset \mathbf{R}^{4 \mid 4}$.

We will consider the twistor space $\mathbf{T}=\mathbf{R}^{4 \mid 4}$; elements of $\mathbf{T}$ are vectors parametrized by even $\lambda_{\dot{\alpha}}, \mu^{\alpha}$ and odd $\zeta^{I}$ :

$$
Z=\left(\mu^{1}, \mu^{2}, \lambda_{\dot{1}}, \lambda_{\dot{2}}, \zeta_{1}, \zeta_{2}, \zeta_{3}, \zeta_{4}\right)^{T}
$$

Here $(\ldots)^{T}$ means transposition; we think of $Z$ as a "column-vector", but write it as a row in Eq. (6) for typographic reasons. Points of $M$ are 2|2planes $L \subset \mathbf{T}$. They can be parametrized by coordinates $x, \theta, \tilde{\theta}, u$ :

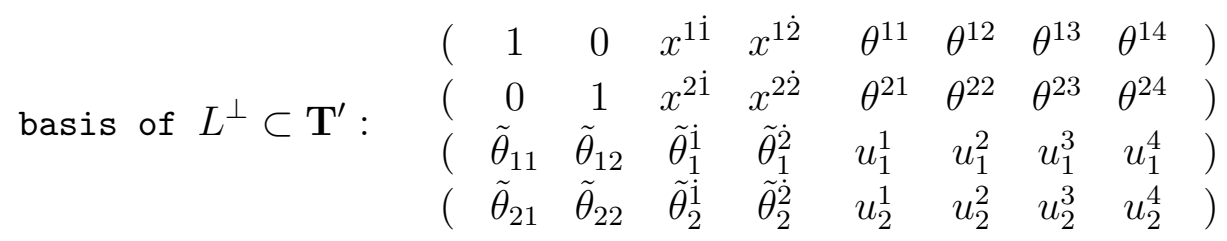

In these coordinates the condition $Z \in L$ is that each row-vector from the set of four vectors (7) has zero scalar product with the column-vector $Z$. These coordinates are redundant. The redundancy can be described as some vector

\footnotetext{
${ }^{4}$ although with our choice of signature we cannot directly use complex geometry; we will replace complex analyticity with some polynomiality condition.
} 
fields whose flows do not change $L$, for example:

$$
\begin{aligned}
& A^{i \alpha}\left(\frac{\partial}{\partial \tilde{\theta}_{i \alpha}}+x^{\alpha \dot{1}} \frac{\partial}{\partial \tilde{\theta}_{i}^{\dot{1}}}+x^{\alpha \dot{2}} \frac{\partial}{\partial \tilde{\theta}_{i}^{\dot{2}}}+\theta^{\alpha I} \frac{\partial}{\partial u_{i}^{I}}\right) \\
& A_{L}^{K}\left(\tilde{\theta}_{K A} \frac{\partial}{\partial \tilde{\theta}_{L A}}+\tilde{\theta}_{K}^{\dot{A}} \frac{\partial}{\partial \tilde{\theta}_{L}^{\dot{A}}}+u_{K}^{I} \frac{\partial}{\partial u_{L}^{I}}\right)
\end{aligned}
$$

for any constant $A^{i \alpha}$ and $A_{L}^{K}$. This can be used to put $\tilde{\theta}_{i \alpha}=0$ :

$$
\tilde{\theta}_{11}=\tilde{\theta}_{12}=\tilde{\theta}_{21}=\tilde{\theta}_{22}=0
$$

To summarize:

$$
\begin{aligned}
\text { twistors } & =\mathbf{T}=\mathbf{R}^{4 \mid 4} \\
\text { space-time } & =M=\operatorname{Gr}(2 \mid 2, \mathbf{T})
\end{aligned}
$$

A special role is played by the 2|2-dimensional tautological vector bundle over $M$ which will be denoted $\mathcal{S}_{M}$. The fiber of $\mathcal{S}_{M}$ over $L \subset M$ is $L$ itself. $\mathcal{S}_{M}$

Also, PT will denote the projective super-twistor space, i.e. $Z$ modulo rescaling: $Z \simeq \kappa Z$.

\subsection{Berezinian and some notations}

For any vector bundle $\mathcal{V}$, we define two line bundles, which we call Ber $\mathcal{V}$ and $|\operatorname{Ber}| \mathcal{V}$. Sections of Ber $\mathcal{V}$ are functions of the bases of the fiber $V$, Ber satisfying the property:

$$
\sigma\left(\left\{m_{I}^{J} e_{J}\right\}\right)=\operatorname{SDet} m \sigma\left(\left\{e_{I}\right\}\right)
$$

where for a supermatrix $m=\left(\begin{array}{cc}A & B \\ C & D\end{array}\right)$ :

$$
\text { SDet } m=\operatorname{det}\left(A-B D^{-1} C\right)(\operatorname{det} D)^{-1}
$$

Similarly, section of $|\operatorname{Ber}| V$ satisfy:

$$
\sigma\left(\left\{m_{I}^{J} e_{J}\right\}\right)=\operatorname{sign}(\operatorname{det} A) \operatorname{SDet} m \sigma\left(\left\{e_{I}\right\}\right)
$$


The characteristic property of $|\operatorname{Ber}| \mathcal{V}$ is that a section of $|\operatorname{Ber}| \mathcal{V}$ defines an operation of integration along the fiber:

$$
\left[\begin{array}{c}
\text { functions on } \mathcal{V} \\
\text { with compact support } \\
\text { on each fiber } V
\end{array}\right] \stackrel{\int}{\longrightarrow}\left[\begin{array}{c}
\text { functions } \\
\text { on the base }
\end{array}\right]
$$

It seems logical to call sections of Ber $\mathcal{V}$ "integral forms on the fiber", and section of $|\operatorname{Ber}| \mathcal{V}$ "volume elements on the fiber".

We will denote $(\operatorname{Ber} \mathcal{V})^{-1}$ the line bundle dual to Ber $\mathcal{V}$, i.e. same as $(\operatorname{Ber} \mathcal{V})^{\prime}$, and $|\operatorname{Ber} \mathcal{V}|^{-1}=|\operatorname{Ber} \mathcal{V}|^{\prime}$.

\subsection{Action of $G L(4 \mid 4, \mathbf{R})$ transformations on twistors}

The twistor vector space $\mathbf{T}$ should be considered a fundamental representation of $G L(4 \mid 4, \mathbf{R})$. We have:

$$
G L(4 \mid 4) / \text { center }=P G L(4 \mid 4)=P S L(4 \mid 4, \mathbf{R}) \rtimes U(1)_{R}
$$

The $u(1)_{R}$ acts as follows:

$$
R Z=\left(\begin{array}{rr}
-\mathbf{1}_{4 \times 4} & \mathbf{0}_{4 \times 4} \\
\mathbf{0}_{4 \times 4} & \mathbf{1}_{4 \times 4}
\end{array}\right) Z
$$

In terms of the parametrization $(6)$ :

$$
R=-\lambda_{\dot{\alpha}} \frac{\partial}{\partial \lambda_{\dot{\alpha}}}-\mu^{\alpha} \frac{\partial}{\partial \mu^{\alpha}}+\zeta^{I} \frac{\partial}{\partial \zeta^{I}}
$$

\subsection{Solutions of free classical super-Maxwell equations from twistor space}

In the free field limit a classical solution of the super-Yang-Mills theory with gauge group $U(N)$ can be obtained as an $N \times N$ matrix whose entries are classical solutions of the super-Maxwell equations. For our purpose it is enough to consider free solutions of the form:

$$
\left[\begin{array}{c}
\text { solution of } \\
\text { super-Maxwell equations }
\end{array}\right] \times\left[\begin{array}{l}
N \times N \\
\text { matrix }
\end{array}\right]
$$


In this Section we will explain the correspondence between solutions of free classical super-Maxwell equations on $M$ and sections of $\left(|\operatorname{Ber}| \mathcal{S}_{M}\right)^{-1}$ which are polynomial in the coordinates $u_{i}^{I}$ defined in Eq. (7). The choice of coordinates $u_{i}^{I}$ is somewhat arbitrary, but the statement of polynomial dependence does not depend on it; in particular this requirement is $P S L(4 \mid 4, \mathbf{R}) \rtimes U(1)_{R^{-}}$ invariant.

Notice that $|\operatorname{Ber}| \mathcal{S}_{M}=\left(|\operatorname{Ber}|\left(\mathbf{T} / \mathcal{S}_{M}\right)\right)^{-1}=|\operatorname{Ber}|\left(\mathbf{T} / \mathcal{S}_{M}\right)^{\prime}=|\operatorname{Ber}| \mathcal{S}_{M}^{\perp}$. Those four vectors listed in Eq. (7) are the basis of $\mathcal{S}_{M}^{\perp}$.

Suppose that we are given a section (here $\Gamma$ (line bundle) denotes the space of sections):

$$
\sigma \in \Gamma\left(\left(|\operatorname{Ber}| \mathcal{S}_{M}\right)^{-1}\right)
$$

which is a polynomial in $u$. The condition that $\sigma$ is annihilated by the vector fields defined in Eq. (9) with $A_{L}^{K}$ satisfying $A_{K}^{K}=0$ implies that it can depend on $\tilde{\theta}$ and $u$ only through the expressions $\tilde{\theta}_{[1}^{\dot{\alpha}} u_{2]}^{I}, u_{[1}^{I} u_{2]}^{J}$ and $\tilde{\theta}_{[1}^{\dot{\alpha}} \tilde{\theta}_{2]}^{\dot{\beta}}$. Since we require $\sigma$ to be a polynomial in $u$, it must be then a polynomial of these three expressions. Moreover, $\sigma$ should have charge 2 under rescaling of $(\tilde{\theta}, u)$, therefore this polynomial is actually a linear function:

$$
\sigma=\tilde{\theta}_{[1}^{\dot{\alpha}} \tilde{\theta}_{2]}^{\dot{\beta}} \mathbf{F}_{\dot{\alpha} \dot{\beta}}^{+}(x, \theta)+\tilde{\theta}_{[1}^{\dot{\alpha}} u_{2]}^{I} \mathbf{\Psi}_{\dot{\alpha} I}(x, \theta)+u_{[1}^{I} u_{2]}^{J} \mathbf{\Phi}_{I J}(x, \theta)
$$

Consider the condition that $\sigma$ does not change when we add to first and second vectors of the basis of Eq. (7) a linear combination of the third and fourth vectors. It implies that $\sigma$ should be annihilated by the following vector fields $V_{i \alpha}$ :

$$
\begin{aligned}
V_{i \alpha} \sigma & =0 \\
\text { where } V_{i \alpha} & =\tilde{\theta}_{i}^{\dot{\beta}} \frac{\partial}{\partial x^{\alpha \dot{\beta}}}+u_{i}^{I} \frac{\partial}{\partial \theta^{\alpha I}}
\end{aligned}
$$

In the rest of this Section, we will first prove that Eq. 23 implies the 
following equations:

$$
\begin{aligned}
\epsilon^{\dot{\gamma} \dot{\beta}} \frac{\partial}{\partial x^{\alpha \dot{\gamma}}} \mathbf{F}_{\dot{\alpha} \dot{\beta}}^{+}(x, \theta) & =0 \\
\frac{\partial}{\partial \theta^{\alpha I}} \mathbf{F}_{\dot{\alpha} \dot{\beta}}^{+}(x, \theta) & =\frac{\partial}{\partial x^{\alpha(\dot{\beta}}} \mathbf{\Psi}_{\dot{\alpha}) I}(x, \theta), \\
\epsilon^{\dot{\alpha} \dot{\beta}} \frac{\partial}{\partial x^{\alpha \dot{\alpha}}} \boldsymbol{\Psi}_{\dot{\beta} I}(x, \theta) & =0 \\
\frac{\partial}{\partial \theta^{\alpha(I}} \boldsymbol{\Psi}_{J) \dot{\alpha}}(x, \theta) & =0 \\
\frac{\partial}{\partial \theta^{\alpha[I}} \boldsymbol{\Psi}_{J] \dot{\alpha}}(x, \theta) & =\frac{\partial}{\partial x^{\alpha \dot{\alpha}}} \boldsymbol{\Phi}_{I J}(x, \theta), \\
\frac{\partial}{\partial \theta^{\alpha P}} \boldsymbol{\Phi}_{I J}(x, \theta) & =\frac{\partial}{\partial \theta^{\alpha[P}} \boldsymbol{\Phi}_{I J]}(x, \theta),
\end{aligned}
$$

and then show that Eqs. (25)- (30) imply that the $\theta$-expansion of $\mathbf{F}_{\dot{\alpha} \dot{\beta}}^{+}(x, \theta), \mathbf{\Psi}_{J \dot{\alpha}}(x, \theta)$ and $\boldsymbol{\Phi}_{I J}(x, \theta)$ can be expressed through solutions of free super-Maxwell theory.

Proof that Eq. (23) implies Eqns. 25) - (30) Consider the expansion of the left hand side of Eq. (23) in powers of $\tilde{\theta}$.

- At the zeroth order in $\tilde{\theta}$, the term that contributes is

$$
u_{m}^{P} u_{[1}^{I} u_{2]}^{J} \frac{\partial}{\partial \theta^{\alpha P}} \boldsymbol{\Phi}_{I J}(x, \theta)
$$

Its vanishing implies Eq. 30

- At the linear order in $\tilde{\theta}$, we have

$$
-u_{m}^{I} \tilde{\theta}_{[1}^{\dot{\alpha}} u_{2]}^{J} \frac{\partial}{\partial \theta^{\alpha I}} \boldsymbol{\Psi}_{\dot{\alpha} J}(x, \theta)+\tilde{\theta}_{m}^{\dot{\beta}} u_{[1}^{I} u_{2]}^{J} \partial_{\alpha \dot{\beta}} \boldsymbol{\Phi}_{I J}(x, \theta),
$$

where $\partial_{\alpha \dot{\beta}}$ denotes $\frac{\partial}{\partial x^{\alpha \dot{\beta}}}$. Notice:

$$
u_{i}^{I} u_{j}^{J}=\epsilon_{i j} u_{[1}^{[I} u_{2]}^{J]}+u_{(i}^{(I} u_{j)}^{J)},
$$

Therefore the terms of Eq. 32 can be written as:

$$
u_{[1}^{I} u_{2]}^{J} \tilde{\theta}_{m}^{\dot{\alpha}}\left(-\frac{\partial}{\partial \theta^{\alpha[I}} \boldsymbol{\Psi}_{J] \dot{\alpha}}(x, \theta)+\partial_{\alpha \dot{\alpha}} \boldsymbol{\Phi}_{I J}(x, \theta)\right)-u_{(1}^{I} u_{2)}^{J} \tilde{\theta}_{m}^{\dot{\alpha}} \frac{\partial}{\partial \theta^{\alpha(I}} \boldsymbol{\Psi}_{J) \dot{\alpha}}(x, \theta) .
$$

The vanishing of this expression implies Eqs. (29) and 28). 
- The terms of the second order in $\tilde{\theta}$ are

$$
u_{m}^{I} \tilde{\theta}_{[1}^{\dot{\alpha}} \tilde{\theta}_{2]}^{\dot{\beta}} \frac{\partial}{\partial \theta^{\alpha I}} \mathbf{F}_{\dot{\alpha} \dot{\beta}}^{+}(x, \theta)+\tilde{\theta}_{m}^{\dot{\beta}} \tilde{\theta}_{[1}^{\dot{\alpha}} u_{2]}^{I} \partial_{\alpha \dot{\beta}} \mathbf{\Psi}_{\dot{\alpha} I}(x, \theta),
$$

using

$$
\tilde{\theta}_{m}^{\dot{\beta}} \tilde{\theta}_{k}^{\dot{\alpha}}=\epsilon^{\dot{\beta}} \dot{\theta^{\prime}} \tilde{\theta}_{(m}^{1} \tilde{\theta}_{k)}^{2]}+\epsilon_{m k} \tilde{\theta}_{[1}^{(\dot{\beta}} \tilde{\theta}_{2]}^{\dot{\alpha})},
$$

Eqn. (35) can be written as

$$
u_{m}^{I} \tilde{\theta}_{[1}^{\dot{\alpha}} \tilde{\theta}_{2]}^{\dot{\beta}}\left(\frac{\partial}{\partial \theta^{\alpha I}} \mathbf{F}_{\dot{\alpha} \dot{\beta}}^{+}(x, \theta)-\partial_{\alpha(\dot{\beta}} \mathbf{\Psi}_{\dot{\alpha}) I}\right)+\tilde{\theta}_{(1}^{[1} \tilde{\theta}_{2)}^{2]} u_{m}^{I}\left(\epsilon^{\dot{\beta} \dot{\alpha}} \partial_{\alpha \dot{\beta}} \mathbf{\Psi}_{\dot{\alpha} I}(x, \theta)\right),
$$

This is only zero when both terms vanish separately, implying Eqs. (26) and (27).

- At third order in $\tilde{\theta}$, the term that contributes is:

$$
T_{\dot{\gamma} \dot{\beta} \dot{\alpha}} \tilde{\theta}_{m}^{\dot{\gamma}} \tilde{\theta}_{[1}^{\dot{\beta}} \tilde{\theta}_{2]}^{\dot{\alpha}},
$$

where $T_{\dot{\gamma} \dot{\beta} \dot{\alpha}}=\partial_{\alpha \dot{\gamma}} \mathbf{F}_{\dot{\alpha} \dot{\beta}}^{+}(x, \theta)$. The vanishing of this expression implies that $T_{\dot{\gamma} \dot{\beta} \dot{\alpha}}$ must be totally symmetric, and therefore Eq. (25).

This concludes the derivation of the constraints (25)- (30) on the superfields $\mathbf{F}_{\dot{\alpha} \dot{\beta}}^{+}(x, \theta), \boldsymbol{\Psi}_{J \dot{\alpha}}(x, \theta)$ and $\boldsymbol{\Phi}_{I J}(x, \theta)$.

Solution to Eqns. 25)- (30) Now we will prove that the components of the $\theta$-expansion of $\mathbf{F}_{\dot{\alpha} \dot{\beta}}^{+}(x, \theta), \mathbf{\Psi}_{J \dot{\alpha}}(x, \theta)$ and $\boldsymbol{\Phi}_{I J}(x, \theta)$ can be expressed through solutions of free super-Maxwell theory.

Eq. (30) implies:

$$
\partial_{\gamma K} \partial_{\beta L} \partial_{M \alpha} \boldsymbol{\Phi}_{I J}(x, \theta)=\partial_{\gamma[K} \partial_{|\beta| L} \partial_{M|\alpha|} \Phi_{I J]}(x, \theta)=0,
$$

which implies that the expansion of $\boldsymbol{\Phi}_{I J}(x, \theta)$ in powers of $\theta$ terminates at the second order:

$$
\boldsymbol{\Phi}_{I J}(x, \theta)=\varphi_{I J}(x)+\epsilon_{I J P Q} \theta^{\alpha P} \tilde{\psi}_{\alpha}^{Q}(x)+\frac{1}{2} \epsilon_{I J P Q} \theta^{\alpha P} \theta^{\beta Q} \tilde{f}_{\alpha \beta}(x) .
$$


Eqns. 28), 29) determine $\boldsymbol{\Psi}_{\dot{\alpha} J}(x, \theta)$. The solution only exists when

$$
\partial_{[\alpha \mid \dot{\alpha}} \tilde{\psi}_{\mid \beta]}^{Q}=0 \text { and } \partial_{[\alpha \mid \dot{\alpha}} \tilde{f}_{\mid \beta] \gamma}=0 .
$$

which are Dirac and Maxwell equations. It is given by the following expression:

$$
\begin{aligned}
\Psi_{J \dot{\alpha}}(x, \theta) & =\psi_{J \dot{\alpha}}(x)+\theta^{\alpha I} \partial_{\alpha \dot{\alpha}} \varphi_{J I}(x)+\frac{1}{2} \epsilon_{J I P Q} \theta^{\alpha I} \theta^{\beta P} \partial_{(\alpha \mid \dot{\alpha}} \tilde{\psi}_{\mid \beta)}^{Q}(x) \\
& +\frac{1}{3 !} \epsilon_{J I P Q} \theta^{\alpha I} \theta^{\beta P} \theta^{\gamma Q} \partial_{(\alpha \mid \dot{\alpha}} \tilde{f}_{\mid \beta \gamma)}(x),
\end{aligned}
$$

Eq. (26) determines $\mathbf{F}_{\dot{\alpha} \dot{\beta}}^{+}(x, \theta)$, but the solution only exists when besides Eqs. (41) also:

$$
\square \varphi_{I J}=0,
$$

It is given by:

$$
\begin{aligned}
\mathbf{F}_{\dot{\alpha} \dot{\beta}}^{+}(x, \theta) & =f_{\dot{\alpha} \dot{\beta}}(x)+\theta^{J \rho} \partial_{\rho \dot{\beta}} \psi_{J \dot{\alpha}}(x)+\frac{1}{2} \theta^{J \rho} \theta^{\alpha I} \partial_{(\rho \mid \dot{\beta}} \partial_{\mid \alpha) \dot{\alpha}} \varphi_{I J}(x) \\
& +\frac{1}{3 !} \epsilon_{J I P Q} \theta^{J \rho} \theta^{\alpha I} \theta^{\beta P} \partial_{(\rho|\dot{\beta}|} \partial_{\alpha|\dot{\alpha}|} \tilde{\psi}_{\beta}^{Q}(x) \\
& +\frac{1}{4 !} \epsilon_{J I P Q} \theta^{J \rho} \theta^{\alpha I} \theta^{\beta P} \theta^{\gamma Q} \partial_{(\rho|\dot{\beta}|} \partial_{\alpha|\dot{\alpha}|} \tilde{f}_{\beta \gamma)}(x) .
\end{aligned}
$$

To conclude, $\sigma$ is given by Eqn. (22) where $\mathbf{F}_{\dot{\alpha} \dot{\beta}}^{+}(x, \theta), \mathbf{\Psi}_{\dot{\beta} J}(x, \theta)$ and $\boldsymbol{\Phi}_{I J}(x, \theta)$ are given by Eqns. (44), (42) and (40), respectively.

\section{Coherent wavefunctions in free theory}

We will now discuss a special family of solutions of free equations, which form an orbit of $P S L(4 \mid 4)$.

For every point $Z \in \mathbf{T}$ we define a generalized function section of $\left(|\operatorname{Ber}| \mathcal{S}_{M}\right)^{-1}$ (see Eq. (21)), which we call $\delta_{Z}$. By definition $\delta_{Z}(X)$, for a fixed $2 \mid 2$ dimensional $X \subset \mathbf{T}$, is the delta-function of the condition that $Z \in X$. More precisely (here $\Gamma$ (line bundle) denotes the space of sections):

$\delta_{Z}(X)$ 


$$
\begin{aligned}
\delta_{Z} \in & \Gamma\left(|\operatorname{Ber}| \mathcal{S}_{M}\right)^{-1} \\
& \text { such that } \forall s \in \Gamma\left(|\operatorname{Ber}| \mathcal{S}_{M}\right): \\
& \int_{Z \in \mathbf{T}} f(Z)\left\langle s(X), \delta_{Z}(X)\right\rangle=\int_{Z \in X} s(X) f(Z),
\end{aligned}
$$

Here the integration on the left hand side uses the canonical measure on $\mathbf{T}$, and the integration measure on the right hand side is the integration along the fiber of $\mathcal{S}_{M}$ defined by the volume element $s$ (Section 2.2). In coordinates (6), (7):

$$
\begin{aligned}
\delta_{Z}(X)= & \left(u_{1}^{I} \zeta_{I}+\tilde{\theta}_{1}^{\dot{\alpha}} \lambda_{\dot{\alpha}}\right)\left(u_{2}^{J} \zeta_{J}+\tilde{\theta}_{2}^{\dot{\alpha}} \lambda_{\dot{\alpha}}\right) \times \\
& \times \delta\left(\mu^{1}+x^{1 \dot{\alpha}} \lambda_{\dot{\alpha}}+\theta^{1 I} \zeta_{I}\right) \delta\left(\mu^{2}+x^{2 \dot{\alpha}} \lambda_{\dot{\alpha}}+\theta^{2 I} \zeta_{I}\right)
\end{aligned}
$$

So defined $\delta_{Z}(X)$, as a function of $X \in M$ (or rather a section of the line bundle $(|\operatorname{Ber}| \mathcal{S})^{-1}$ over $\left.M\right)$, encodes a solution of $\mathcal{N}=4$ super-Maxwell equations of motion as described in Section 2.

To determine the corresponding scalar, electromagnetic and spinor fields, we write $\delta_{Z}(X)$ as a sum of expressions with definite values of the $R$ charge, which corresponds to twice the power of $\zeta$ :

$$
\begin{aligned}
\tilde{\theta}_{1}^{\dot{\alpha}} \tilde{\theta}_{2}^{\dot{\beta}} f_{\dot{\alpha} \dot{\beta}} & : \tilde{\theta}_{1}^{\dot{\alpha}} \tilde{\theta}_{2}^{\dot{\beta}} \lambda_{\dot{\alpha}} \lambda_{\dot{\beta}} \delta^{2}(\mu+x \lambda) \\
u_{[1}^{J} \tilde{\theta}_{2]}^{\dot{\alpha}} \psi_{\dot{\alpha} J} & : \tilde{\theta}_{[1}^{\dot{\alpha}} \lambda_{\dot{\alpha}} u_{2]}^{J} \zeta_{J} \delta^{2}(\mu+x \lambda) \\
u_{1}^{I} u_{2}^{J} \varphi_{I J} & : u_{1}^{I} u_{2}^{J} \zeta_{I} \zeta_{J} \delta^{2}(\mu+x \lambda) \\
u_{1}^{[I} u_{2}^{J} \theta^{K] \alpha} \tilde{\psi}_{I J K \alpha} & : u_{1}^{[I} u_{2}^{J} \theta^{K] \alpha} \zeta_{I} \zeta_{J} \zeta_{K} \partial_{\alpha} \delta^{2}(\mu+x \lambda) \\
u_{1}^{[I} u_{2}^{J} \theta^{K \alpha} \theta^{L] \beta} \tilde{f}_{\alpha \beta} & : u_{1}^{[I} u_{2}^{J} \theta^{K \alpha} \theta^{L] \beta} \zeta_{I} \zeta_{J} \zeta_{K} \zeta_{L} \partial_{\alpha} \partial_{\beta} \delta^{2}(\mu+x \lambda)
\end{aligned}
$$

\section{Beta deformation of the free Yang-Mills ac- tion}

We want to characterize a deforming operator $\int d^{4} x U$ in Eq. (1) by its value on free solutions. In particular, in this paper we are interested in the case of beta-deformation, which corresponds to deforming the action by an expression cubic in the elementary fields [13. Let us evaluate the corresponding $U$ on the formal sum of three coherent states:

$$
\left(\varepsilon_{1} \delta_{Z_{(1)}}(X)+\varepsilon_{2} \delta_{Z_{(2)}}(X)+\varepsilon_{3} \delta_{Z_{(3)}}(X)\right) M
$$


with bosonic nilpotent coefficients $5 \varepsilon_{1}, \varepsilon_{2}, \varepsilon_{3}$, where $M$ is some $N \times N$-matrix (see Eq. (20)). As we explained in Section 1, linearized beta-deformations are parametrized by $B \in(\mathbf{g} \wedge \mathbf{g})_{0} / \mathbf{g}$. We consider the case when $B$ is a decomposable tensor, i.e. is of the form $B=\xi \wedge \xi$ where $\xi$ is an odd element $\xi \wedge \xi$ of $\mathbf{g}$. The condition of zero internal commutator (the subindex 0 in $\left.(\mathbf{g} \wedge \mathbf{g})_{0}\right)$ means that $\xi$ should be nilpotent. We claim that the result of evaluation of $\int d^{4} x U$ on the free solution given by Eq. 52 is:

$$
\int d^{4} x U=\varepsilon_{1} \varepsilon_{2} \varepsilon_{3} \delta_{Z_{(1)}, Z_{(2)}}^{\xi}\left(Z_{(3)}\right) \operatorname{tr} M^{3}
$$

where $\delta_{Z_{(1)}, Z_{(2)}}^{\xi}\left(Z_{(3)}\right)$ is defined by Eq. (57). In fact, our Eq. 57 works for any nilpotent $\xi \in \Pi T_{e} P S L(4 \mid 4, \mathbf{R})$; this includes odd elements of $\operatorname{psl}(4 \mid 4, \mathbf{R})$ as well as linear combinations of even elements of $\operatorname{psl}(4 \mid 4, \mathbf{R})$ with Grassmann odd coefficient:6.

(At the same time, on the string theory side the integrated vertex operator of the worldsheet sigma-model for this particular $B$ is [9]:

$$
U_{\mathrm{AdS}}=\int j_{a} \xi^{a} \wedge j_{b} \xi^{b}
$$

Here $j_{a}$ is the Noether current on the worldsheet, a one-form.)

\subsection{Definition of $\delta_{Z_{(1)}, Z_{(2)}}^{\xi}(Z)$}

Let us pick two points 7 in PT: $\left[Z_{(1)}\right]$ and $\left[Z_{(2)}\right]$. Let $\xi \in \mathbf{g}$ be an odd nilpotent element of $\mathbf{g}=\operatorname{psl}(4 \mid 4, \mathbf{R})$. We can represent $\xi$ as a $4|4 \times 4| 4$ -

\footnotetext{
5 "Nilpotent" means satisfying $\varepsilon_{1}^{2}=\varepsilon_{2}^{2}=\varepsilon_{3}^{2}=0$. To the best of our knowledge, the idea to use nilpotent coefficient was first suggested in [21]. The authors of 21] constructed a solution of classical field equations, which they called "perturbiner". Our use of nilpotent coefficients is slightly different; we take a solution of the free field equations in the form of Eq. (52) and evaluate some $\int d^{4} x U$ on it. We need the nilpotence of coefficients to avoid considering the square of delta-function.

${ }^{6}$ Since we work with coherent states, we are into supergeometry/supermanifolds; we have to use a "pool" of constant Grassmann odd parameters [22].

${ }^{7}$ Strictly speaking, it is not appropriate to think about "points of a supermanifold". It is better to say that, for an arbitrary supermanifold $S$, we pick two arbitary morphisms $Z_{(1)}: S \rightarrow \mathbf{P T}$ and $Z_{(2)}: S \rightarrow \mathbf{P T}\left[23\right.$. For our purposes, it is enough to take $S=\mathbf{R}^{0 \mid K}$ for large enough $K$. Technically, we just allow all our twistors to depend on $K$ constant Grassmann odd parameters.
} 
supermatrix, which we also denote $\xi$. Since $\xi$ is nilpotent as an element of $\operatorname{psl}(4 \mid 4, \mathbf{R})$, the square of this matrix is proportional to the unit matrix:

$$
\xi^{2}=c \mathbf{1}
$$

where $c$ is some number. We assume that $\xi$ is nondegenerate, in the following sense: either $c \neq 0$, or if $c=0$ then $\operatorname{Ker} \xi=\operatorname{Im} \xi$. Let us consider a $2 \mid 2$ dimensional plane $L$ generated by $Z_{(1)}, Z_{(2)}, \xi Z_{(1)}$ and $\xi Z_{(2)}$ :

$$
L=\mathbf{R} Z_{(1)}+\mathbf{R} Z_{(2)}+\mathbf{R} \xi Z_{(1)}+\mathbf{R} \xi Z_{(2)}
$$

Let us define $\delta_{Z_{(1)}, Z_{(2)}}^{\xi}(Z)$, essentially a delta-function of $Z$, in the following way. For any test function $f \in C^{\infty}(\mathbf{T})$ :

$$
\begin{aligned}
& \int_{Z \in \mathbf{R}^{4 \mid 4}} \delta_{Z_{(1)}, Z_{(2)}}^{\xi}(Z) f(Z)= \\
= & \int_{\mathbf{R}^{2}} d a_{1} \wedge d a_{2} \frac{\partial}{\partial \psi^{1}} \frac{\partial}{\partial \psi^{2}} f\left(a_{1} Z_{(1)}+a_{2} Z_{(2)}+\psi^{1} \xi Z_{(1)}+\psi^{2} \xi Z_{(2)}\right)
\end{aligned}
$$

The integration on the left hand side uses the canonical measure on $\mathbf{R}^{4 \mid 4}$ (the " $S$ " in "PSL(4|4)"). The integral on the right hand side uses the integral form on $L$ canonically defined by $\xi$ as described in Appendix A.

Eq. (57) implies that $\delta_{Z_{(1)}, Z_{(2)}}^{\xi}(Z)$ is a linear function of $\xi \otimes \xi$. It has weight zero in $Z$ and also in both $Z_{(1)}$ and $Z_{(2)}$; in other words, for $\kappa \in \mathbf{R}$ :

$$
\delta_{Z_{(1)}, Z_{(2)}}^{\xi}(\kappa Z)=\delta_{\kappa Z_{(1)}, Z_{(2)}}^{\xi}(Z)=\delta_{Z_{(1)}, \kappa Z_{(2)}}^{\xi}(Z)=\delta_{Z_{(1)}, Z_{(2)}}^{\xi}(Z)
$$

Equivalently, we can define $\delta_{Z_{(1)}, Z_{(2)}}^{\xi}(Z)$ as follows:

$$
\begin{aligned}
& \delta_{Z_{(1)}, Z_{(2)}}^{\xi}(Z)= \\
= & \int_{\mathbf{R}^{2}} d a_{1} \wedge d a_{2} \frac{\partial}{\partial \psi^{1}} \frac{\partial}{\partial \psi^{2}} \delta^{(4 \mid 4)}\left(Z-a_{1} Z_{(1)}-a_{2} Z_{(2)}-\psi^{1} \xi Z_{(1)}-\psi^{2} \xi Z_{(2)}\right)
\end{aligned}
$$

\subsection{Orientation of $L_{\mathrm{rd}}$ and discontinuity of $\delta_{Z_{(1)}, Z_{(2)}}^{\xi}$}

In order to integrate an integral form:

$$
d a_{1} \wedge d a_{2} \frac{\partial}{\partial \psi^{1}} \frac{\partial}{\partial \psi^{2}} f\left(a_{1} Z_{(1)}+a_{2} Z_{(2)}+\psi^{1} \xi Z_{(1)}+\psi^{2} \xi Z_{(2)}\right)
$$


over a supermanifold $L$ (Eq. (56)), we need an orientation of its body $L_{\mathrm{rd}}=\mathbf{R}^{2}$. This $\mathbf{R}^{2}$ is generated by $Z_{(1)}$ and $Z_{(2)}$. Therefore, we need to know which basis has positive orientation: $\left\{Z_{(1)}, Z_{(2)}\right\}$ or $\left\{Z_{(2)}, Z_{(1)}\right\}$ ? A comparison with explicit calculation in Section 4.5 shows that the orientation is determined by the sign of $\epsilon^{\dot{\alpha} \dot{\beta}} \lambda_{(1) \dot{\alpha}} \lambda_{(2) \dot{\beta}}$. This sounds like a contradiction, because $\epsilon^{\dot{\alpha} \dot{\beta}} \lambda_{(1) \dot{\alpha}} \lambda_{(2) \dot{\beta}}$ is not conformally invariant. However, the sign of $\epsilon^{\dot{\alpha} \dot{\beta}} \lambda_{(1) \dot{\alpha}} \lambda_{(2) \dot{\beta}}$ is invariant under infinitesimal conformal transformations. Explicit calculation in Section 4.5 shows that $\delta_{Z_{(1)}, Z_{(2)}}^{\xi}(Z)$, as a function of $Z_{(1)}$ and $Z_{(2)}$, has a discontinuity when $\epsilon^{\dot{\alpha} \dot{\beta}} \lambda_{(1) \dot{\alpha}} \lambda_{(2) \dot{\beta}}=0$. The limit at the discontinuity is proportional to $\delta^{(2)}\left(\lambda-a_{1} \lambda_{(1)}-a_{2} \lambda_{(2)}\right)$, but the sign in front of $\delta^{(2)}\left(\lambda-a_{1} \lambda_{(1)}-a_{2} \lambda_{(2)}\right)$ depends on whether we are approaching from $\epsilon^{\dot{\alpha} \dot{\beta}} \lambda_{(1) \dot{\alpha}} \lambda_{(2) \dot{\beta}}>0$ or from $\epsilon^{\dot{\alpha} \dot{\beta}} \lambda_{(1) \dot{\alpha}} \lambda_{(2) \dot{\beta}}<0$.

This can be explained as follows. We interpret the four-dimensional spacetime as the real Grassmannian: $\mathbf{R}^{2,2} \cup \infty=\operatorname{Gr}(2,4)$ (Section 2). For the Yang-Mills action to be conformally invariant, the scalar field should transform as a section of $(\operatorname{Ber} S)^{-1}$, and spinors as sections of $S \otimes(\operatorname{Ber} S)^{-1}$ (chiral) and $S^{\perp} \otimes(\operatorname{Ber} S)^{-1}$ (antichiral), where $S$ is the tautological vector bundle of $\operatorname{Gr}(2,4)$ (like $\mathcal{S}_{M}$ of Section 2.1 but without "super-"). However, the $\delta_{Z}$ of Section 3 gives sections of $(\ldots) \otimes|\operatorname{Ber}|^{-1}$ instead of $(\ldots) \otimes \operatorname{Ber}^{-1}$ (Section 2.2 . We would be able to cast sections of $\mid$ Ber $\left.\right|^{-1}$ as sections of $\mathrm{Ber}^{-1}$, if we provided an orientation of $S$. But this is only possible locally. In fact, we can provide orientation of the fiber of $S$ over all $x$ except at infinity. This is acceptable for our computation, because the integral $\int d^{4} x U$ in Eq. (1) is supported on a single point $x \in \mathbf{R}^{2,2}$ determined by the intersection of the planes $\mu_{(1)}-\lambda_{(1)} x=0$ and $\mu_{(2)}-\lambda_{(2)} x=0$. But when we consider the case when $\lambda_{(1)} \rightarrow \lambda_{(2)}$, the intersection point $x$ goes to $\infty$, where the orientation is undefined, essentially because $\|x\|^{2}=\operatorname{det} x$ can be positive or negative depending on how $x \rightarrow \infty$. This leads to the discontinuity of $\delta_{Z_{(1)}, Z_{(2)}}^{\xi}(Z)$.

\subsection{Symmetry of $\delta_{Z_{(1)}, Z_{(2)}}^{\xi}(Z)$}

The defining Eq. 57 implies that $\delta_{Z_{(1)}, Z_{(2)}}^{\xi}(Z)$ is symmetric under the exchange $Z_{(1)} \leftrightarrow Z_{(2)}$. In fact it is symmetric under arbitrary permutations of 
$Z, Z_{(1)}, Z_{(2)}$. This can be proven as follows:

$$
\begin{aligned}
& \delta^{(4 \mid 4)}\left(Z-a_{1} Z_{(1)}-a_{2} Z_{(2)}-\psi_{1} \xi Z_{(1)}-\psi_{2} \xi Z_{(2)}\right)= \\
= & \operatorname{sign}\left(a_{1}\right) \delta^{(4 \mid 4)}\left(\frac{1}{a_{1}+\psi_{1} \xi} Z-Z_{(1)}-\frac{a_{2}}{a_{1}+\psi_{1} \xi} Z_{(2)}-\frac{1}{a_{1}+\psi_{1} \xi} \psi_{2} \xi Z_{(2)}\right)= \\
= & \operatorname{sign}\left(a_{1}\right) \times \\
& \times \delta^{(4 \mid 4)}\left(-Z_{(1)}+a_{1}^{-1} Z-\left(a_{1}^{-1} a_{2}-a_{1}^{-2} \psi_{1} \psi_{2} c\right) Z_{(2)}-a_{1}^{-2} \psi_{1} \xi Z-\right. \\
& \left.\quad-\left(a_{1}^{-1} \psi_{2}-a_{1}^{-2} a_{2} \psi_{1}\right) \xi Z_{(2)}\right)
\end{aligned}
$$

The change of variables:

$$
\begin{aligned}
& \tilde{a}_{1}=a_{1}^{-1} \\
& \tilde{a}_{2}=-a_{1}^{-1} a_{2}+a_{1}^{-2} \psi_{1} \psi_{2} c \\
& \tilde{\psi}_{1}=-a_{1}^{-2} \psi_{1} \\
& \tilde{\psi}_{2}=a_{1}^{-2} a_{2} \psi_{1}-a_{1}^{-1} \psi_{2}
\end{aligned}
$$

in the integral $\int_{\mathbf{R}^{2}} d a_{1} \wedge d a_{2} \frac{\partial}{\partial \psi^{1}} \frac{\partial}{\partial \psi^{2}}$ is equivalent to the exchang $\rfloor^{8} Z \leftrightarrow Z_{1}$.

\subsection{The case when $\xi \wedge \xi$ corresponds to zero deforma- tion}

Suppose that $\xi \wedge \xi$ belongs to the denominator of Eq. (5):

$$
\xi \wedge \xi=\Delta(\zeta)=\sum C^{a b} t_{a} \wedge\left[t_{b}, \zeta\right]
$$

for some $\zeta \in \operatorname{psl}(4 \mid 4)$, and $C^{a b}$ the inverse of the Killing metric. We know from [9] that such $\xi \wedge \xi$ should correspond to zero deformations. We will now prove that for such $\xi$ Eq. (57) indeed evaluates to zero.

Let us enumerate the basis vectors of $\mathbf{T}:\left\{e_{1}, e_{2}, e_{3}, e_{4}, f_{1}, f_{2}, f_{3}, f_{4}\right\}$ where $e$ are even and $f$ are odd. Without loss of generality, let us assume that $Z_{(1)}=e_{1}$ and $Z_{(2)}=e_{2}$. (In other words, we choose a basis so that the first two elements are $Z_{(1)}$ and $Z_{(2)}$.)

\footnotetext{
${ }^{8}$ The sign factor $\operatorname{sign}\left(a_{1}\right)$ is compensated by the possible difference of sign between $\epsilon^{\dot{\alpha} \dot{\beta}} \lambda_{(1) \dot{\alpha}} \lambda_{(2) \dot{\beta}}$ and $\epsilon^{\dot{\alpha} \dot{\beta}} \lambda_{\dot{\alpha}} \lambda_{(2) \dot{\beta}}$. This sign determines the orientation of $\mathbf{R}^{2}$ which is needed to integrate $d a_{1} \wedge d a_{2}$.
} 
Let us first consider the case when $\zeta$ is even:

$$
\zeta=e_{a} \otimes e^{\vee b}-\frac{1}{4} \delta_{a}^{b} \mathbf{1}
$$

where $e^{\vee}, f^{\vee}$ are elements of the dual basis and $\mathbf{1}=\sum_{c} e_{c} \otimes e^{\vee c}$. In this case:

$$
\Delta(\zeta)=\sum_{c=1}^{4}\left(e_{a} \otimes e^{\vee c}-\frac{1}{4} \delta_{a}^{c} \mathbf{1}\right) \wedge\left(e_{c} \otimes e^{\vee b}-\frac{1}{4} \delta_{c}^{b} \mathbf{1}\right)+\text { odd } \wedge \text { odd }
$$

The odd $\wedge$ odd terms do not contribute to the computation. Also, the subtractions do not contribute to the computation, because the integral of Eq. (59):

$$
\int_{\mathbf{R}^{2}} d a^{1} \wedge d a^{2} \frac{\partial}{\partial \psi^{1}} \frac{\partial}{\partial \psi^{2}} f\left(a^{1} Z_{(1)}+a^{2} Z_{(2)}+\psi^{1} \xi_{1} Z_{(1)}+\psi^{2} \xi_{2} Z_{(2)}\right)
$$

is zero when either $\xi_{1}$ or $\xi_{2}$ is proportional to $\mathbf{1}$ (for example, when $\xi_{1}=\alpha \mathbf{1}$, the dependence on $\psi^{1}$ can be removed by shifting $\left.a^{1} \mapsto a^{1}-\psi^{1} \alpha\right)$. Let us pick a pair of fermionic constants $\epsilon$ and $\eta$ from the pool[22]; we are evaluating:

$$
\begin{aligned}
\int_{\mathbf{R}^{2}} d a_{1} \wedge d a_{2} \frac{\partial}{\partial \psi^{1}} \frac{\partial}{\partial \psi^{2}} \sum_{c=1}^{4} f( & a^{1} e_{1}+a^{2} e_{2}+ \\
& \left.+\psi^{1}\left(\epsilon \delta_{1}^{c} e_{a}+\eta \delta_{1}^{b} e_{c}\right)+\psi^{2}\left(\epsilon \delta_{2}^{c} e_{a}+\eta \delta_{2}^{b} e_{c}\right)\right)
\end{aligned}
$$

This integral is equal to zero. In fact, each term in $\sum_{c=1}^{4}$ vanishes separately. Indeed, when $c$ is 3 or 4 it is proportional to $\eta^{2}=0$. If $c$ is 1 or 2 , we can shift the integration variable $a^{1}$ or $a^{2}$ to eliminate the dependence on $\eta$; then the integral becomes proportional to $\epsilon^{2}=0$.

Now consider the case when $\zeta$ is odd:

$$
\begin{aligned}
\zeta & =f_{a} \otimes e^{\vee b} \\
\Delta(\zeta) & =\left(f_{a} \otimes e^{\vee c}\right) \wedge\left(e_{c} \otimes e^{\vee b}-\frac{1}{4} \delta_{c}^{b} \mathbf{1}\right)+\left(f_{a} \otimes f^{\vee c}-\frac{1}{4} \delta_{a}^{c} \mathbf{1}\right) \wedge\left(f_{c} \otimes e^{\vee b}\right)
\end{aligned}
$$


Again, the second term does not contribute to the computation. The contribution of the first term is:

$$
\begin{aligned}
\int_{\mathbf{R}^{2}} d a_{1} \wedge d a_{2} \frac{\partial}{\partial \psi^{1}} \frac{\partial}{\partial \psi^{2}} \sum_{c} f & \left(a^{1} e_{1}+a^{2} e_{2}+\right. \\
& \left.+\psi^{1}\left(\delta_{1}^{c} f_{a}+\eta \delta_{1}^{b} e_{c}\right)+\psi^{2}\left(\delta_{2}^{c} f_{a}+\eta \delta_{2}^{b} e_{c}\right)\right)
\end{aligned}
$$

The terms with $c$ equal 3 or 4 are zero, being proportional to $\eta^{2}=0$. When $c$ is 1 or 2 , we can eliminate the dependence on $\eta$ by shifting $a$, and then we are left with, for example, when $c=1$ :

$$
\int_{\mathbf{R}^{2}} d a_{1} \wedge d a_{2} \frac{\partial}{\partial \psi^{1}} \frac{\partial}{\partial \psi^{2}} \sum_{c} f\left(a^{1} e_{1}+a^{2} e_{2}+\psi^{1} f_{a}\right)
$$

This integral is zero, because the integrand does not depend on $\psi^{2}$.

\subsection{Deformation of the form $\int \phi \psi \psi$}

To prove our claim that the evaluation of the deforming operator on the free solution given by Eq. 52 is indeed $\delta_{Z_{(1)}, Z_{(2)}}^{\xi}(Z)$, we will consider a particular case when the test function $f(Z)$ of Eq. (57) is:

$$
f(Z)=A_{I K}(\lambda, \mu) \epsilon^{I K P Q} \zeta_{P} \zeta_{Q}
$$

Eq. 53 implies that evaluation of $\int_{Z \in \mathbf{R}^{4 \mid 4}} \delta_{Z_{(1)}, Z_{(2)}}^{\xi}(Z) f(Z)$ with such $f(Z)$ corresponds to the substitution instead of the term proportional to $\varepsilon_{3}$ in Eq. (52) of the following solution of the linearized equations of motion (cp. Eq. (49)):

$$
\begin{aligned}
& \varphi_{I K}(x)=\int d^{2} \lambda d^{2} \mu A_{I K}(\lambda, \mu) \delta^{2}(\mu+x \lambda) \\
& \psi=\tilde{\psi}=F^{+}=F^{-}=0
\end{aligned}
$$

Let us choose both $\xi_{1}$ and $\xi_{2}$ as "rotations of $S^{5}$ ", i.e.:

$$
\left(\xi_{1} \wedge \xi_{2}\right)_{K L}^{I J}=B_{K L}^{I J}
$$

with other components of $\xi_{1} \wedge \xi_{2}$ all zero. 
Eq. (57) implies that $\int_{Z \in \mathbf{R}^{4 \mid 4}} \delta_{Z_{(1)}, Z_{(2)}}^{\xi}(Z) f(Z)$ equals to:

$$
\int_{\mathbf{R}^{2}} A_{P Q}\left(a^{(1)} \lambda_{(1)}+a^{(2)} \lambda_{(2)}, a^{(1)} \mu_{(1)}+a^{(2)} \mu_{(2)}\right) \epsilon^{P Q R S} B_{R S}^{I J} \zeta_{(1) I} \zeta_{(2) J} d a^{(1)} \wedge d a^{(2)}
$$

The same result is obtained by evaluating the deformation term in the Lagrangian [13], using the formulas of Appendix B:

$$
\begin{aligned}
& \int d^{4} x \epsilon^{I J K L} \varphi_{I J}(x) B_{K L}^{P Q} \psi_{(1) P \dot{\alpha}}(x) \psi_{(2) Q \dot{\beta}}(x) \epsilon^{\dot{\alpha} \dot{\beta}} \\
& \text { where } \psi_{(A) \dot{\alpha} K}(x)=\lambda_{(A) \dot{\alpha}} \zeta_{(A) K} \delta^{2}\left(\mu_{(A)}+x \lambda_{(A)}\right)
\end{aligned}
$$

This establishes the contact with the description of the supermultiplet on the field theory side obtained in [13]; matching of other states follows from applying supersymmetry ${ }^{9}$.

Fourier transform into the usual momentum space We will use now the notations of [14]. Following the prescription in [14], let us substitute in Eq. (78):

$$
A_{I K}(\lambda, \mu)=\delta^{2}\left(\lambda-\lambda_{(0)}\right) \exp \left(i \mu \tilde{\lambda}_{(0)}\right) \zeta_{(0) I} \zeta_{(0) K}
$$

and then multiply by the Fourier transform factor $\exp \left(-i \mu_{(1)} \tilde{\lambda}_{(1)}-i \mu_{(2)} \tilde{\lambda}_{(2)}\right)$ and integrate over $\mu_{(1)}$ and $\mu_{(2)}$. Then Eq. (81) gives:

$$
\begin{aligned}
\int d a^{1} \wedge d a^{2} & \delta^{2}\left(\lambda_{(0)}-a^{(1)} \lambda_{(1)}-a^{(2)} \lambda_{(2)}\right) \times \\
& \times \delta^{2}\left(\tilde{\lambda}_{(1)}-a^{(1)} \tilde{\lambda}_{(0)}\right) \delta^{2}\left(\tilde{\lambda}_{(2)}-a^{(2)} \tilde{\lambda}_{(0)}\right) \zeta_{(0) P} \zeta_{(0) Q} \epsilon^{P Q R S} B_{R S}^{I J} \zeta_{(1) I} \zeta_{(2) J}
\end{aligned}
$$

Using Appendix B, this is equal to:

$$
\begin{aligned}
& \frac{1}{\left\langle\lambda_{(1)}, \lambda_{(2)}\right\rangle} \delta^{2}\left(\tilde{\lambda}_{(1)}-\frac{\left\langle\lambda_{(2)}, \lambda_{(0)}\right\rangle}{\left\langle\lambda_{(2)}, \lambda_{(1)}\right\rangle} \tilde{\lambda}_{(0)}\right) \delta^{2}\left(\tilde{\lambda}_{(2)}-\frac{\left\langle\lambda_{(1)}, \lambda_{(0)}\right\rangle}{\left\langle\lambda_{(1)}, \lambda_{(2)}\right\rangle} \tilde{\lambda}_{(0)}\right) \times \\
& \times \zeta_{(0) P} \zeta_{(0) Q} \epsilon^{P Q R S} B_{R S}^{I J} \zeta_{(1) I} \zeta_{(2) J}
\end{aligned}
$$

\footnotetext{
${ }^{9}$ Vanishing of deformations corresponding to the denominator in Eq. (5) was not proven in [13. It follows from Section 4.4 that such deformations would vanish when evaluated on (52). This should imply that the deformation is acually zero, although we do not have a rigorous proof.
} 
Remember [14] that the momenta of the scattering particles are $p_{(i) \alpha \dot{\alpha}}=$ $\tilde{\lambda}_{(i) \alpha} \lambda_{(i) \dot{\alpha}}$. Eq. 86 can be interpreted as a deformation of the three-point scattering amplitude. It is a generalized function with support on $\tilde{\lambda}_{(1)}, \tilde{\lambda}_{(2)}$ and $\tilde{\lambda}_{(0)}$ being all collinear to each other.

\section{Other finite-dimensional representations}

It is likely that the expressions $\delta_{Z_{1}, Z_{2}}^{\xi}(Z)$ will serve as building block for evaluation of deformations corresponding to other finite-dimensional representations. It was conjectured in [4] that deformations of the form:

$$
\begin{aligned}
& \int d^{4} x \rho_{0}(x) \operatorname{tr}\left(\Phi_{1}+i \Phi_{2}\right)^{4}(x), \int d^{4} x \rho_{1}(x) \operatorname{tr}\left(\Phi_{1}+i \Phi_{2}\right)^{5}(x), \\
& \int d^{4} x \rho_{2}(x) \operatorname{tr}\left(\Phi_{1}+i \Phi_{2}\right)^{6}(x), \quad \ldots
\end{aligned}
$$

where $\rho_{n}$ are some special polynomial functions described in [4] generate finite-dimensional representations of $\mathbf{g}$ corresponding to supersymmetric Young diagramms:
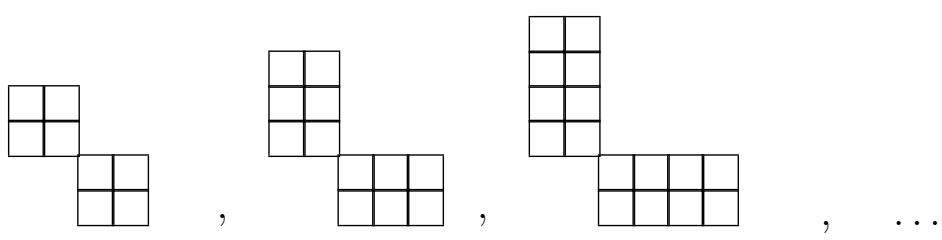

and their transposed. An element of such a representation is a super-traceless tensor $B$ with $2(n+2)$ lower indices and $2(n+2)$ upper indices. The symmetry type of the lower indices is determined by the lower portion of the Young diagramms, and of the upper indices by the upper portion. For example, the

Young diagramm $\quad \forall$ ("case $n=-1$ ") corresponds to the tensors of the form $B_{c d}^{a b}$ symmetric in $a b$ and antysymmetric in $c d$, while $\square$ corresponds to the tensors of the form $B_{c d}^{a b}$ antisymmetric in $a b$ and symmetric in $c d$.

When all indices $a, b, c, d$ are fermionic, $\square$ gives $B_{K L}^{I J}=B_{K L}^{J I}=-B_{L K}^{I J}$ (see Eq. (82).

Consider the tensor $B=\xi^{\otimes 2(n+2)}$ where $\xi$ is an odd nilpotent element of g. Let us apply the Young symmetrizer corresponding to: 


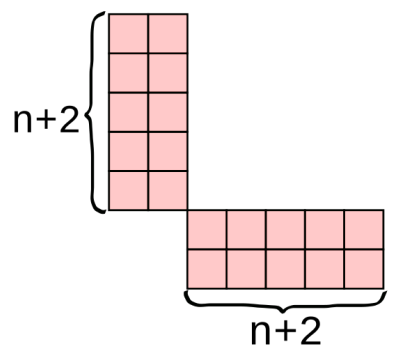

We conjecture that the evaluation of the deformation on the coherent state (as in Eq. 20):

$$
\left(\varepsilon_{1} \delta_{Z_{1}}(X)+\varepsilon_{2} \delta_{Z_{2}}(X)+\ldots+\varepsilon_{n+4} \delta_{Z_{n+4}}(X)\right) M
$$

gives $\delta_{Z_{1}, \ldots, Z_{n+4}}^{\xi} \operatorname{tr} M^{n+4}$ where:

$$
\delta_{Z_{1}, \ldots, Z_{n+4}}^{\xi}=\delta_{Z_{1}, Z_{2}}^{\xi}\left(Z_{3}\right) \delta_{Z_{2}, Z_{3}}^{\xi}\left(Z_{4}\right) \cdots \delta_{Z_{n+2}, Z_{n+3}}^{\xi}\left(Z_{n+4}\right)
$$

We leave the verification of this statement for future work. The symmetry of $\delta_{Z_{1}, \ldots, Z_{n}}^{\xi}$ under permutations of $Z_{i}$ can be proven as in Section 4.3 .

Let us prove that Eq. (91) depends on $\xi \wedge \cdots \wedge \xi$ only through its Young projector. To start, notice that each $\delta_{Z_{n}, Z_{n+1}}^{\xi}\left(Z_{n+2}\right)$ depends on $\xi \wedge \xi$ via the projector $\square_{\xi \otimes \xi}$. (In other words, the lower through $\xi_{[c}^{(a} \xi_{d]}^{b)}$.) This follows from the fact that $\delta_{Z_{n}, Z_{n+1}}^{\xi}\left(Z_{n+2}\right)=0$ when $\xi$ is degenerate, $\operatorname{dim} \operatorname{im} \xi=1$ (this follows from the definition Eq. (57)). This implies that $\delta_{Z_{1}, \ldots, Z_{n+4}}^{\xi}$ depends on $\xi \wedge \cdots \wedge \xi$ only through:

$$
\xi_{\left[c_{1}\right.}^{\left(a_{1}\right.} \xi_{\left.d_{1}\right]}^{\left.b_{1}\right)} \xi_{\left[c_{2}\right.}^{\left(a_{2}\right.} \xi_{\left.d_{2}\right]}^{\left.b_{2}\right)} \cdots \xi_{\left[c_{n+2}\right.}^{\left(a_{n+2}\right.} \xi_{\left.d_{n+2}\right]}^{\left.b_{n+2}\right)}
$$

But this is not all, there are more projectors. Let us consider the special case $n=0$. Let us write $\delta_{Z_{1}, Z_{2}, Z_{3}, Z_{4}}^{\xi}$ as $\delta_{Z_{2}, Z_{3}}^{\xi}\left(Z_{1}\right) \delta_{Z_{2}, Z_{3}}^{\xi}\left(Z_{4}\right)$. The indices $c_{1}$ and $c_{2}$ both contract with $Z_{2}$, therefore they enter symmetrized. The indices $d_{1}$ and $d_{2}$ also enter symmetrized, because they both contract with $Z_{3}$. Therefore,

the $\xi \wedge \xi \wedge \xi \wedge \xi$ enters only through the projector to $\boxplus$. In general, $\xi^{\wedge 2(n+2)}$ enters only through the projector of Eq. 89 ).

The second diagramm of Eq. (3) can be probed by replaying our construction with $\mathbf{T}$ replaced with $\Pi \mathbf{T}$ (the twistor space with flipped statistics). 


\section{Acknowledgments}

The authors are partially supported by the FAPESP grant 2014/18634-9 "Dualidade Gravitação/Teoria de Gauge". The work of A.M. was also supported in part by the RFBR grant 15-01-99504 "String theory and integrable systems". The work of S.P.M was supported by the CNPq grant $154704 / 2014-8$.

\section{A Measure defined by an odd linear operator}

A nondegenerate odd nilpotent linear operator $\xi$ on an $n \mid n$-dimensional linear space defines an integration measure, in the following way. Given any n-tuple of even vectors $Z_{1}, \ldots, Z_{n}$ we define the integral of an arbitrary function $f$ as follows:

$$
\int f=\int_{\mathbf{R}^{n \mid n}} d a^{1} \cdots d a^{n} \frac{\partial}{\partial \psi^{1}} \cdots \frac{\partial}{\partial \psi^{n}} f\left(a^{i} Z_{i}+\psi^{i} \xi Z_{i}\right)
$$

In fact, it is not strictly necessary that $\xi$ is nilpotent; it is enough that the square of $\xi$ be proportional to a unit matrix:

$$
\xi^{2}=c \mathbf{1}
$$

where $c$ is some number. All we have to prove that the definition given by Eq. (93) is independent of the choice of $Z_{1}, \ldots, Z_{n}$. Suppose that we choose a different $n$-tuple:

$$
\widetilde{Z}_{i}=Z_{i}+\epsilon_{i}^{j} \xi Z_{j}
$$

We replace $Z$ with $\widetilde{Z}$ in Eq. 93 ; the integrand becomes:

$$
\begin{aligned}
f\left(a^{i} Z_{i}+\psi^{i} \xi Z_{i}\right) & =f\left(a^{i} Z_{i}+a^{i} \epsilon_{i}^{j} \xi Z_{j}+\psi^{i} \xi Z_{i}+\psi^{i} \epsilon_{i}^{j} c Z_{j}\right)= \\
& =f\left(a^{i} Z_{i}+\left(\psi^{i}+a^{j} \epsilon_{j}^{i}\right) \xi Z_{i}+\left(\psi^{i}+a^{j} \epsilon_{j}^{i}\right) \epsilon_{i}^{k} c Z_{k}-a^{j} \epsilon_{j}^{k} \epsilon_{k}^{i} c Z_{i}\right)
\end{aligned}
$$

The difference between Eq. (96) and the integrand of Eq. (93) can be undone by the change of variables $\widetilde{\psi}^{i}=\psi^{i}+a^{j} \epsilon_{j}^{i}, \widetilde{a}^{i}=a^{i}-a^{j} \epsilon_{j}^{k} \epsilon_{k}^{i} c$. (Notice that the determinant of the change from $a$ to $\widetilde{a}$ is 1 , since $\epsilon_{j}^{i}$ are fermionic.) 


\section{B Some integrals}

We denote:

$$
\left\langle\lambda_{1}, \lambda_{2}\right\rangle=\epsilon^{\dot{\alpha} \dot{\beta}} \lambda_{1 \dot{\alpha}} \lambda_{2 \dot{\beta}} \quad, \quad\left\langle\mu_{1}, \mu_{2}\right\rangle=\epsilon_{\alpha \beta} \mu^{1 \alpha} \mu^{2 \beta}
$$

To integrate a 2-form $d a^{1} \wedge d a^{2}$, we need an orientation of the $\left(a_{1}, a_{2}\right)$-plane. We orient it as $\left(a_{1}, a_{2}\right)$ if $\left\langle\lambda_{1}, \lambda_{2}\right\rangle>0$ and as $\left(a_{2}, a_{1}\right)$ otherwize. Then we get:

$$
\left\langle\lambda_{1}, \lambda_{2}\right\rangle \int d a^{1} \wedge d a^{2} \delta^{2}\left(\lambda-a^{1} \lambda_{1}-a^{2} \lambda_{2}\right)=1
$$

Therefore:

$$
\begin{aligned}
& \int d^{4} x \delta^{2}(\mu+x \lambda)\left\langle\lambda_{1}, \lambda_{2}\right\rangle \delta^{2}\left(\mu_{1}+x \lambda_{1}\right) \delta^{2}\left(\mu_{2}+x \lambda_{2}\right)= \\
= & \left\langle\lambda_{1}, \lambda_{2}\right\rangle^{2} \int d a^{1} \wedge d a^{2} \delta^{2}\left(\lambda-a^{1} \lambda_{1}-a^{2} \lambda_{2}\right) \times \\
& \times \int d^{4} x \delta^{2}(\mu+x \lambda) \delta^{2}\left(\mu_{1}+x \lambda_{1}\right) \delta^{2}\left(\mu_{2}+x \lambda_{2}\right)= \\
= & \int d a^{1} \wedge d a^{2} \delta^{2}\left(\lambda-a^{1} \lambda_{1}-a^{2} \lambda_{2}\right) \delta^{2}\left(\mu-a^{1} \mu_{1}-a^{2} \mu_{2}\right)= \\
= & \left\langle\lambda_{1}, \lambda_{2}\right\rangle \delta^{2}\left(\left\langle\lambda_{1}, \lambda_{2}\right\rangle \mu-\left\langle\lambda, \lambda_{2}\right\rangle \mu_{1}+\left\langle\lambda, \lambda_{1}\right\rangle \mu_{2}\right)= \\
= & \left\langle\lambda_{1}, \lambda_{2}\right\rangle\left|\left\langle\mu_{1}, \mu_{2}\right\rangle\right| \delta\left(\left\langle\lambda_{1}, \lambda_{2}\right\rangle\left\langle\mu, \mu_{1}\right\rangle-\left\langle\mu_{1}, \mu_{2}\right\rangle\left\langle\lambda, \lambda_{1}\right\rangle\right) \times \\
& \times \delta\left(\left\langle\lambda_{1}, \lambda_{2}\right\rangle\left\langle\mu, \mu_{2}\right\rangle-\left\langle\mu_{1}, \mu_{2}\right\rangle\left\langle\lambda, \lambda_{2}\right\rangle\right)
\end{aligned}
$$

\section{References}

[1] O. Aharony, S. S. Gubser, J. M. Maldacena, H. Ooguri, and Y. Oz, Large $N$ field theories, string theory and gravity, Phys. Rept. 323 (2000) 183-386 doi: 10.1016/S0370-1573(99)00083-6 [arXiv/hep-th/9905111].

[2] M. Gunaydin, D. Minic, and M. Zagermann, 4D doubleton conformal theories, CPT and IIB string on $A d S_{5} \times S^{5}$, Nucl. Phys. B534 (1998) 96-120 doi: 10.1016/S0550-3213(98)00801-3, 10.1016/S0550-3213(98)00543-4 [arXiv/hep-th/9806042]. [Erratum: Nucl. Phys.B538,531(1999)]. 
[3] A. Mikhailov, Symmetries of massless vertex operators in $A d S(5) x$ $S^{* * 5}$, Adv. Theor.Math.Phys. 15 (2011) 1319-1372 doi:

10.4310/ATMP.2011.v15.n5.a3.

[4] A. Mikhailov, Finite dimensional vertex, JHEP 1112 (2011) 5 doi: 10.1007/JHEP12(2011)005 [arXiv/1105.2231].

[5] N. Berkovits and T. Fleury, Harmonic Superspace from the $A d S_{5} \times S^{5}$ Pure Spinor Formalism, JHEP 03 (2013) 022 doi:

10.1007/JHEP03(2013)022 [arXiv/1212.3296].

[6] A. Mikhailov and R. Xu, BRST cohomology of the sum of two pure spinors, arXiv/1301.3353.

[7] N. Berkovits, Super Poincare covariant quantization of the superstring, JHEP 04 (2000) 018 doi: 10.1088/1126-6708/2000/04/018 [arXiv/hep-th/0001035].

[8] R. G. Leigh and M. J. Strassler, Exactly marginal operators and duality in four-dimensional $N=1$ supersymmetric gauge theory, Nucl. Phys.

B447 (1995) 95-136 doi: 10.1016/0550-3213(95)00261-P [arXiv/hep-th/9503121].

[9] O. A. Bedoya, L. Bevilaqua, A. Mikhailov, and V. O. Rivelles, Notes on beta-deformations of the pure spinor superstring in $A d S(5) \times S(5)$, Nucl.Phys. B848 (2011) 155-215 doi:

10.1016/j.nuclphysb.2011.02.012 [arXiv/1005.0049].

[10] A. Mikhailov, Cornering the unphysical vertex, JHEP 082 (2012) doi: 10.1007/JHEP11(2012)082 [arXiv/1203.0677].

[11] A. Mikhailov, Vertex operators of ghost number three in Type IIB supergravity, Nucl. Phys. B907 (2016) 509-541 doi:

10.1016/j.nuclphysb.2016.04.007 [arXiv/1401.3783].

[12] L. Wulff and A. A. Tseytlin, Kappa-symmetry of superstring sigma model and generalized 10d supergravity equations, JHEP 06 (2016) 174 doi: 10.1007/JHEP06(2016)174 [arXiv/1605.04884].

[13] S. P. Milian, Supermultiplet of $\beta$-deformations from twistors, arXiv/1607.06506. 
[14] E. Witten, Perturbative gauge theory as a string theory in twistor space, Commun.Math.Phys. 252 (2004) 189-258 doi:

10.1007/s00220-004-1187-3 [arXiv/hep-th/0312171].

[15] N. Berkovits, An Alternative string theory in twistor space for $N=4$ superYang-Mills, Phys. Rev. Lett. 93 (2004) 011601 doi:

10.1103/PhysRevLett.93.011601 [arXiv/hep-th/0402045].

[16] M. Kulaxizi and K. Zoubos, Marginal deformations of N=4 SYM from open/closed twistor strings, Nucl. Phys. B738 (2006) 317-349 doi:

10.1016/j.nuclphysb.2006.01.018 [arXiv/hep-th/0410122].

[17] M. Kulaxizi and K. Zoubos, Marginal deformations of tree-level $N=4$ SYM from twistor string theory, Fortsch. Phys. 54 (2006) 423-429 doi: 10.1002/prop.200510299 [arXiv/hep-th/0512302].

[18] P. Gao and J.-B. Wu, (Non)-supersymmetric marginal deformations from twistor string theory, Nucl. Phys. B798 (2008) 184-197 doi: 10.1016/j.nuclphysb.2008.01.027 [arXiv/hep-th/0611128].

[19] H. Elvang, D. Z. Freedman, and M. Kiermaier, A simple approach to counterterms in $N=8$ supergravity, JHEP 11 (2010) 016 doi:

10.1007/JHEP11(2010)016 [arXiv/1003.5018].

[20] Y. I. Manin, Gauge field theory and complex geometry, vol. 289 of Grundlehren der Mathematischen Wissenschaften [Fundamental Principles of Mathematical Sciences]. Springer-Verlag, Berlin, second ed., 1997. Translated from the 1984 Russian original by N. Koblitz and J. R. King, With an appendix by Sergei Merkulov.

[21] A. A. Rosly and K. G. Selivanov, On amplitudes in self-dual sector of Yang-Mills theory, Phys. Lett. B399 (1997) 135-140 hep-th/9611101.

[22] A. S. Shvarts, On the Definition of Superspace, Theor. Math. Phys. 60 (1984) 657-660 doi: 10.1007/BF01018248. [Teor. Mat.

Fiz.60,37(1984)].

[23] J. Bernstein, Lectures on supersymmetry, . 